4. Légaré JF, Ross DB, Issekutz TB, Ruigrok W, Creaser K, Hirsch GM, et al. Prevention of allograft heart valve failure in a rat model. J Thorac Cardiovasc Surg. 2001; 122:310-7.

5. Légaré JF, Lee TD, Creaser K, Ross DB. T lymphocytes mediate leaflet destruction and allograft aortic valve failure in rats. Ann Thorac Surg. 2000;70:1238-45.

6. Cebotari S, Mertsching H, Kallenbach K, Kostin S, Repin O, Batrinac A, et al. Construction of autologous human heart valves based on an acellular allograft matrix. Circulation. 2002;106(12 Suppl 1):I63-8.

doi:10.1016/S0022-5223(03)00597-X

\section{Obstruction of St Jude Medical valves in the aortic position}

\section{To the Editor:}

I enjoyed the recent article by Teshima and coworkers, "Obstruction of St Jude Medical Valves in the Aortic Position: Histology and Immunohistochemistry of Pannus" (J Thorac Cardiovasc Surg. 2003;126:401-

$7)$. I recently reoperated on 2 young female patients with dysfunction of St Jude Medical prosthetic valves (St Jude Medical, Inc, St Paul, Minn) in the aortic position. Both patients had moderate amounts of pannus formation on the valve. Both patients had significant periods during their postoperative management when anticoagulation was discontinued or inadequate. Perhaps more significantly, they had small aortic roots. The explanted St Jude Medical valves were $19 \mathrm{~mm}$ in both patients.

Are young female patients who receive 19- or 21-mm implants for small aortic roots predisposed toward pannus formation? Is pannus formation detected only in 19- and 21-mm valves, in which a minor reduction in orifice area may result in clinically apparent hemodynamic deterioration? I noticed the female predilection in the published series and wondered about the size of the explanted prostheses and if the postimplantation coagulation history of these patients was known.

Frank A. Baciewicz, Jr, MD Department of Cardiothoracic Surgery Harper Hospital

Wayne State University

Detroit, MI 48201

doi:10.1016/j.jtcvs.2003.11.066

\section{Reply to the Editor:}

We read with interest the letter by Dr Baciewicz regarding his experience of two young female patients with prosthetic valve dysfunction as a result of pannus formation. In our study, we showed histologic and immunohistochemical findings of pannus tissue that caused prosthetic valve dysfunction after aortic valve replacement. We believe that our study provides information useful to elucidate the mechanisms of pannus formation in patients receiving prosthetic valves. We also think, however, that our findings are not sufficient to reach conclusion regarding the complete pathogenetic mechanisms of this disorder, and further prospective studies including more patients and control specimens are required.

The sizes of the prosthesis explanted in this study were $21 \mathrm{~mm}$ in 6 patients (all female), $23 \mathrm{~mm}$ in 2 ( 1 male and 1 female), $25 \mathrm{~mm}$ in 1 (male), and $27 \mathrm{~mm}$ in 1 (male). The ratio of effective orifice area (according to the manufacturer's report) to body surface area, as an index of patient-prosthesis mismatch, in our patients with pannus receiving $21-\mathrm{mm}$ prostheses was $1.0 \pm$ $0.1 \mathrm{~cm}^{2}$. The ratio was slightly lower than that in patients with normally functioning 21-mm aortic prostheses $\left(1.2 \pm 0.2 \mathrm{~cm}^{2}\right){ }^{1}$ Because remaining significant transvalvular gradient after valve replacement may induce shear stress at the periannular tissue, patient-prosthesis mismatch per se can be a cause of excessive intimal growth. Another important issue in patients with small aortic annuli is mechanical contact between the ventricular septum and the pivot guard of the prosthesis. It is conceivable that the pivot guard protrudes to the left ventricular side, which is unique to the design of St Jude Medical valves, ${ }^{2}$ and thus is readily in contact with the hypertrophied ventricular septum. Recently, we carefully examined patients with prosthetic valve dysfunction in the aortic position with multidetector row computed tomography. ${ }^{3}$ In that study, these pathognomic conditions and pannus formation located mainly on the pivot guard of the septal side were confirmed. Therefore the possibility exists that patients with small aortic annuli are predisposed toward pannus formation regardless of the absence of patient-prosthesis mismatch, especially when the patient has a small left ventricular outflow tract.

During the period of this study, 615 patients underwent aortic valve replacement with St Jude Medical valves, and 265 of those were female (43\%). Therefore, as indicated by Dr Baciewicz, the female proportion of patients who had prosthetic valve dysfunction develop tended to be higher than that of patients with normal prosthetic valve function. A major reason for this appears to be simply that female patients had smaller aortic annulus diameters. The mean age of our 7 female patients who developed pannus formation was 62.6 \pm 6.7 years, which did not differ from our 265 female patients with normally functioning aortic valve prostheses. Although the number of patients is small and our results do not have statistical power, patient age per se may not be an important factor in pannus formation.

In our study, 4 of 11 patients with prosthetic valve dysfunction had a short-term history of warfarin withdrawal during treatments for noncardiac disease (abdominal operations and dental treatment). The remaining 7 patients received adequate anticoagulation therapy with warfarin throughout the postoperative period. The observations during reoperations and histologic examinations of periannular tissue did not demonstrate thrombus in all patients. These findings, however, do not negate the presence of perivalvular thrombus at an earlier period of prosthetic valve dysfunction. Because pannus can develop as a result of prosthetic valve thrombosis, inadequate anticoagulation also is considered to be a risk factor for this disorder.

The cause of prosthetic valve dysfunction related to pannus may be multifactorial, and definitive mechanisms for this disorder have not yet been conclusively shown. On the basis of our findings that excessive expression of transforming growth factor $\beta$ was observed in the pannus, however, chronic inflammatory reaction in the periannular lesions-perhaps as a result of surgical trauma, reaction to the foreign materials, mechanical contact, inadequate anticoagulation, and shear stress at the annulus-may be associated with pannus formation.

Hideki Teshima, MD Nobuhiko Hayashida, MD Shigeaki Aoyagi, MD

Department of Surgery Kurume University 67 Asahi-machi Kurume, Japan 


\section{References}

1. Aoyagi S, Nishimi Y, Kawano H, Tayama E, Fukunaga S, Hayashida N, et al. Obstruction of St. Jude Medical valves in the aortic position: significance of a combination of cineradiography and echocardiography. $J$ Thorac Cardiovasc Surg. 2000;120:142-7.

2. Aoyagi S, Nishimi Y, Tayama E, Fukunaga $\mathrm{S}$, Hayashida N, Akashi H, et al. Obstruction of St. Jude Medical valves in the aortic position: a consideration for pathogenic mechanism of prosthetic valve obstruction. J Cardiovasc Surg. 2002;10:339-44.

3. Teshima H, Hayashida N, Enomoto N, Aoyagi S, Okuda K, Uchida M. Diagnosis of prosthetic valve dysfunction by multidetector-row computed tomography. Ann Thorac Surg. 2003;75:1631-3.

doi:10.1016/j.jtcvs.2003.11.067

\section{Prosthesis size and prosthesis- patient size are unrelated to prosthesis-patient mismatch \\ To the Editor:}

We read with interest the report of Blackstone and colleagues ${ }^{1}$ published in the September 2003 issue of the Journal, as well as the editorial of Gillinov and coworkers ${ }^{2}$ in the August 2003 issue. The implicit conclusion of these two articles was that prosthesis-patient mismatch (PPM) is a rare occurrence after aortic valve replacement and that it has a negligible impact with regard to postoperative outcomes. To define PPM and analyze its consequences, both sets of authors chose, however, to use an indexed area based on the internal geometric dimension of the prosthesis divided by the patient's body surface area, rather than the indexed effective orifice area, which is the physiologic parameter most often used to define PPM. They justified their choice of parameter on the basis that geometric measures "are determined before implantation, have much less variability, and are independent of hemodynamic state." 1

The physiologic and clinical relevance of the indexed internal geometric area as used by these authors must, however, be challenged. Indeed, it has never been shown that this parameter can be related, in any significant manner, to transvalvular pressure gradients; in particular, it has been demonstrated that the indexed internal geometric orifice area cannot be used to predict which patients will have high postoperative gradients. ${ }^{3}$ Inherent to the pathophysiology of valve PPM is the concept that too small a prosthesis in too large a patient will produce abnormally high gradients and thus have potentially detrimental consequences such as might occur with a native aortic stenosis. Thus if the indexed internal geometric area cannot be related to postoperative gradients, we do not see how it can logically be used to identify PPM or to characterize its severity.

In contrast, and despite its inherent limitations, the indexed effective orifice area is the only parameter that has consistently been shown to correlate with postoperative gradients, as well as being highly predictive of adverse outcomes. ${ }^{3-5}$ Indeed, when the definition of PPM is based on this parameter, the phenomenon has been shown to be highly prevalent (19\%-70\%, depending on series ${ }^{4,5}$ ) and to be associated with less symptomatic improvement, worse hemodynamics at rest and during exercise, less regression of left ventricular hypertrophy, and more cardiac events after operation. ${ }^{4,5}$ A recent report from our own laboratory has clearly shown that PPM has a major impact on early mortality, particularly in patients with poor left ventricular function and that, in contrast to other risk factors, it can easily be prevented by use of a simple strategy at the time of operation. ${ }^{5}$ Such a strategy was recently used by Castro and associates ${ }^{6}$; as a result, the incidence of moderate-severe PPM in their population was only $2.5 \%$, instead of the $17 \%$ that would have occurred had this prospective strategy not been used, whereas operative mortality remained low $(1 \%)$. Extrapolating these findings to the total number of aortic valve replacements being performed each year in North America, it is estimated that approximately 1000 operative deaths could potentially be avoided through use of such a strategy.

In this context, the conclusion of these two articles with regard to the prevalence of PPM and its consequences cannot be accepted at face value, because the parameter used to define PPM is not valid to characterize postoperative hemodynamics. ${ }^{3}$ To the contrary, we still believe in the "value of concentrating on better hemodynamic performance"1 and that research aimed at properly identifying PPM, as well as preventing it, can significantly contribute to improved outcomes after aortic valve surgery.

\author{
Jean G. Dumesnil, MD, FRCPC, FACC \\ Philippe Pibarot, DVM, PhD, FACC \\ Research Center of Laval Hospital/Quebec \\ Heart Institute \\ Laval University \\ Sainte-Foy, Quebec, Canada
}

\section{References}

1. Blackstone EH, Cosgrove DM, Jamieson WR, Birkmeyer NJ, Lemmer JH, Miller DC, et al. Prosthesis size and long-term survival after aortic valve replacement. $J$ Thorac Cardiovasc Surg. 2003;126:783-96.

2. Gillinov AM, Blackstone EH, Rodriguez LL. Prosthesis-patient size: measurement and clinical implications. J Thorac Cardiovasc Surg. 2003;126:313-6.

3. Pibarot P, Dumesnil JG, Cartier PC, Métras J, Lemieux M. Patient-prosthesis mismatch can be predicted at the time of operation. Ann Thorac Surg. 2001;71(Suppl 5):S265-8.

4. Pibarot P, Dumesnil JG. Hemodynamic and clinical impact of prosthesis-patient mismatch in the aortic valve position and its prevention. J Am Coll Cardiol. 2000;36: 1131-41.

5. Blais C, Dumesnil JG, Baillot R, Simard S, Doyle D, Pibarot P. Impact of prosthesispatient mismatch on short-term mortality after aortic valvereplacement. Circulation. 2003; 108:983-8.

6. Castro LJ, Arcidi JM, Fisher AL, Gaudiani VA. Routine enlargement of the small aortic root: a preventive strategy to minimize mismatch. Ann Thorac Surg. 2002;74:31-6. doi:10.1016/j.jtcvs.2003.11.073

\section{Reply to the Editor:}

We have long respected the excellent and prolific work of Dumesnil and Pibarot, so it is not surprising that we referred to their publications in our articles. However, they are unhappy in part with the way we have interpreted (or misinterpreted) their work, ${ }^{1,2}$ and in part with the surgical recommendations we based on survival data from our multicenter meta-analysis, ${ }^{1}$ which differ from their own, which were based largely on functional prosthesis performance data.

\section{What We Studied}

\section{Survival}

We studied the relation of geometric prosthesis size to time-related survival with nine sources of data and nearly 70,000 patient-years of follow-up among 13,258 patients who underwent aortic valve replacement with mechanical prostheses, stent- 